INPLASY

PROTOCOL

To cite: Li et al. Efficacy and Safety of Bushen Zhuangjin Decoction in the Treatment of Knee Osteoarthritis: A

Systematic Review and MetaAnalysis. Inplasy protocol

202210118. doi:

10.37766/inplasy2022.1.0118

Received: 24 January 2022

Published: 24 January 2022

Corresponding author:

Wenhao Li

18800152886@163.com

Author Affiliation:

Dongzhimen Hospital Affiliated

to Beijing University of

Chinese Medicine.

Support: No financial support.

Review Stage at time of this

submission: Data analysis.

Conflicts of interest:

None declared.

\section{Efficacy and Safety of Bushen Zhuangjin Decoction in the Treatment of Knee Osteoarthritis: A Systematic Review and Meta-Analysis}

Li, WH1; Niu, YQ2; Xiong, ZC3; Zhao, H4; Li, CH5; Liu, T6; Guan, JB7 ; Yang, YD; Yu, $X^{9}$.

Review question / Objective: To evaluate the efficacy and safety of Bushen Zhuangjin Decoction(BZD) in the treatment of KOA.

Eligibility criteria: All studies included in this meta-analysis met the following criteria: (1) published RCTs; (2) patients with definite diagnosis of KOA, regardless of age, gender and nationality; (3) complete comparative data between the two groups can be obtained by using BZD as intervention and other drugs as control measures.

INPLASY registration number: This protocol was registered with the International Platform of Registered Systematic Review and Meta-Analysis Protocols (INPLASY) on 24 January 2022 and was last updated on 24 January 2022 (registration number INPLASY202210118).

\section{INTRODUCTION}

Review question / Objective: To evaluate the efficacy and safety of Bushen Zhuangjin Decoction(BZD) in the treatment of KOA.

Condition being studied: BZD is a traditional Chinese medicine decoction commonly used in the treatment of KOA, and its efficacy has been confirmed by some studies.

\section{METHODS}

Participant or population: Patients with definite diagnosis of KOA, regardless of age, gender and nationality.

Intervention: BZD. 
Comparator: Glucosamine capsules, NSAIDs, sodium hyaluronate intra-articular injection.

Study designs to be included: Randomized controlled trials.

Eligibility criteria: All studies included in this meta-analysis met the following criteria: (1) published RCTs; (2) patients with definite diagnosis of KOA, regardless of age, gender and nationality; (3) complete comparative data between the two groups can be obtained by using BZD as intervention and other drugs as control measures.

Information sources: PubMed, Embase, Cochrane Library, China National Knowledge Infrastructure database (CNKI), WanFang database, and Chinese Scientific Journal Database.

Main outcome(s): VAS score, WOMAC score, excellent and good rate of curative effect, total effective rate.

Quality assessment / Risk of bias analysis: This study used the Cochrane risk bias tool for quality evaluation. This tool includes evaluations in seven aspects: random sequence generation, allocation hiding, blinding of participants and implementers, blinding of outcome evaluators, incomplete outcome data, selective reporting, and other biases. The risk of bias in each area is judged as low risk, high risk, or unknown risk.

Strategy of data synthesis: The Review Manager software (RevMan 5.3) was used for statistical analysis. Continuous variables were reported as mean difference (MD) and $95 \%$ confidence interval (CI), while dichotomy variables were reported as odds ratio (OR) and $95 \% \mathrm{Cl}$. Statistical heterogeneity was judged by the combination of $Q$ value statistics and 12 statistics. The larger the 12 , the greater the heterogeneity. If there was heterogeneity in the study ( $12 \geq 50 \%$ ), the random effects model was adopted; otherwise, the fixed effects model was adopted $(12<50 \%)$. The extracted data was input into the computer, reviewed, and independently analyzed by two researchers.

Subgroup analysis: Subgroup analyses were performed for BZD and glucosamine capsules, NSAIDs, and sodium hyaluronate, respectively.

Sensitivity analysis: Not yet completed

Country(ies) involved: China.

Keywords: Bushen Zhuangjin Decoction, knee osteoarthritis, meta-analysis.

Contributions of each author:

Author 1 - Wenhao Li.

Author 2 - Yiqun Niu.

Author 3 - Zhencheng Xiong.

Author 4 - He Zhao.

Author 5 - Chuanhong Li.

Author 6 - Tao Liu.

Author 7 - Jianbin Guan.

Author 8 - Yongdong Yang.

Author 9 - Xing Yu. 